\title{
Octet baryon magnetic moments in light cone QCD sum rules
}

\author{
T. M. Aliev ${ }^{a} *$, A. Özpineci ${ }^{b}$, M. Savc1 ${ }^{a} \ddagger$ \\ a Physics Department, Middle East Technical University, 06531 Ankara, Turkey \\ b The Abdus Salam International Center for Theoretical Physics, I-34100, Trieste, Italy
}

\begin{abstract}
Octet baryon magnetic moments are calculated in framework of the light cone QCD sum rules. The analysis is carried for the general form of the interpolating currents for octet baryons. A comparison of our results on the magnetic moments of octet baryons with the predictions of other approaches and experimental data is presented.
\end{abstract}

PACS number(s): 11.55.Hx, 13.40.Em, 14.20.Jn

*e-mail: taliev@metu.edu.tr

${ }^{\dagger}$ e-mail: ozpineci@ictp.trieste.it

‡e-mail: savci@metu.edu.tr 


\section{Introduction}

The method of QCD sum rule [1] has become a powerful tool in studying hadron physics on the basis of QCD. It is a framework which connects physical parameters of hadrons to the QCD parameters. In this approach hadrons are represented by their interpolating quark currents taken at large virtualities and then correlation function of these quark currents is introduced. Following this, on one side, the correlation function is calculated by the operator product expansion (OPE), and on the other side, its phenomenological part is constructed. Physical quantities of interest are determined by matching these two descriptions at large virtualities via dispersion relations.

The QCD sum rule method is discussed in many review articles (see [2]- [6] and references therein) and successfully applied in studying various characteristics of hadrons. One of the important characteristic static parameters of hadrons is their magnetic moments. Magnetic moments of nucleon were calculated in framework of the QCD sum rule approach in [7, 8] using external field technique. They were later refined and extended to the entire baryon octet [9, 10].

In this work, we present an independent calculation of the magnetic moments of the octet baryons in the framework of an alternative approach to the traditional sum rules method, i.e., the light cone QCD sum rules method (LCQSR). In the LCQSR method OPE is carried out near the light cone $x^{2}=0$ instead of at the short distance $x \approx 0$. This is an expansion over the twists of the operators rather than the dimensions, as is the case in the traditional QCD sum rules. The nonperturbative dynamics is parametrized by the so called light cone wave functions, rather than the vacuum condensates used in the traditional QCD sum rules. A detailed description of this method can be found in [6, 11, 12]. There are many applications of the LCQSR in the current literature. Note that magnetic moments of the nucleon and decuplet baryons were studied in [13] and [14], respectively, using the LCQSR method.

The paper is organized as follows. In section 2 the LCQSR for the magnetic moments of the octet baryons are derived. In section 3 we present our numerical calculations and a comparison of the prediction of the LCQSR method on the magnetic moments of the octet baryons with the results of the other methods and experimental results.

\section{LCQSR for the magnetic moments of the octet baryons}

As we have already noted, in order to construct the sum rules for the magnetic moments of hadrons, it is necessary to introduce the correlation function of interpolating quark currents which have the same quantum numbers as corresponding hadrons. For this aim we consider the following correlator function:

$$
\Pi=i \int d^{4} x e^{i p x}\left\langle 0\left|T\left\{\eta_{B}(x) \bar{\eta}_{B}(0)\right\}\right| 0\right\rangle_{\gamma},
$$

where $\gamma$ means the external electromagnetic field, $\eta_{B}$ is the interpolating current of the corresponding baryon. It follows from this expression that we need the explicit expression of the interpolating currents to calculate the correlation function. It is well known that 
there is a continuum number of interpolating currents for the baryons. The general form of the interpolating currents for the octet baryons can be represented as [15, 16]

$$
\begin{aligned}
\eta_{p} & =2 \epsilon_{a b c} \sum_{\ell=1}^{2}\left(u^{a T} C A_{1}^{\ell} d^{B}\right) A_{2}^{\ell} u^{c}, \\
\eta_{n} & =n_{p}\left(u^{c} \rightarrow d^{c}\right), \\
\eta_{\Sigma^{0}} & =\frac{1}{\sqrt{2}} \epsilon_{a b c} \sum_{\ell=1}^{2}\left[\left(u^{a T} C A_{1}^{\ell} s^{b}\right) A_{2}^{\ell} d^{c}-\left(d^{c T} C A_{1}^{\ell} s^{b}\right) A_{2}^{\ell} u^{a}\right], \\
\eta_{\Lambda} & =\frac{2}{\sqrt{6}} \epsilon_{a b c} \sum_{\ell=1}^{2}\left[-2\left(u^{a T} C A_{1}^{\ell} d^{b}\right) A_{2}^{\ell} s^{c}+\left(u^{a T} C A_{1}^{\ell} s^{b}\right) A_{2}^{\ell} d^{c}+\left(d^{c T} C A_{1}^{\ell} s^{b}\right) A_{2}^{\ell} u^{a}\right], \\
\eta_{\Xi^{0}} & =-2 \epsilon_{a b c} \sum_{\ell=1}^{2}\left(s^{a T} C A_{1}^{\ell} u^{b}\right) A_{2}^{\ell} s^{c}, \\
\eta_{\Sigma^{+}} & =\eta_{\Sigma^{0}}(d \rightarrow u), \\
\eta_{\Sigma^{-}} & =\eta_{\Sigma^{0}}(u \rightarrow d), \\
\eta_{\Xi^{-}} & =\eta_{\Xi^{0}}(u \rightarrow d),
\end{aligned}
$$

where $a, b, c$ are the color indices, $A_{1}^{1}=1, A_{1}^{2}=t \gamma_{5}, A_{2}^{1}=\gamma_{5}, A_{2}^{2}=t$ and $t$ is an arbitrary parameter. Ioffe current corresponds to the choice $t=-1$.

Let us firstly discuss the hadronic representation of the correlator function. By inserting a complete set of states between the currents in Eq. (1) with quantum numbers of the corresponding baryon $B$, one obtains the hadronic representation of the correlator

$$
\begin{aligned}
\Pi & =\frac{\left\langle 0\left|\eta_{B}\right| B\left(p_{1}\right)\right\rangle}{p_{1}^{2}-m_{B}^{2}}\left\langle B\left(p_{1}\right) \mid B\left(p_{2}\right)\right\rangle_{\gamma} \frac{\left\langle B\left(p_{2}\right)\left|\bar{\eta}_{B}\right| 0\right\rangle}{p_{2}^{2}-m_{B}^{2}} \\
& +\sum_{h} \frac{\left\langle 0\left|\eta_{B}\right| h\left(p_{1}\right)\right\rangle}{p_{1}^{2}-m_{h}^{2}}\left\langle h\left(p_{1}\right) \mid h\left(p_{2}\right)\right\rangle_{\gamma} \frac{\left\langle h\left(p_{2}\right)\left|\bar{\eta}_{B}\right| 0\right\rangle}{p_{2}^{2}-m_{h}^{2}}
\end{aligned}
$$

where $p_{2}=p_{1}+q$, and $q$ is the photon momentum. The second term in Eq. (3) takes into account higher states and continuum contributions and $h$ forms a complete set of baryons having the same quantum number as the ground state baryon $B$ (here $B$ represents any one of $p, n, \Sigma, \Lambda$ and $\Xi$ ). The coupling strength of the interpolating currents with the baryon states parametrized with the overlap amplitudes $\lambda_{B}$ is defined as

$$
\left\langle 0\left|\eta_{B}\right| B(p)\right\rangle=\lambda_{B} u_{B}(p)
$$

It follows from Eq. (3) that in order to obtain an expression for the phenomenological part of the correlator, explicit form of the matrix element $\left\langle B\left(p_{1}\right) \mid B\left(p_{2}\right)\right\rangle_{\gamma}$ is needed. Electromagnetic vertex of the spin $1 / 2$ baryons can be written as

$$
\begin{aligned}
\left\langle B\left(p_{1}\right) \mid B\left(p_{2}\right)\right\rangle_{\gamma} & =\bar{u}_{B}\left(p_{1}\right)\left[f_{1} \gamma_{\mu}+\frac{i \sigma_{\mu \nu} q^{\nu}}{2 m_{B}} f_{2}\right] u_{B}\left(p_{2}\right) \varepsilon^{\mu} \\
& =\bar{u}_{B}\left(p_{1}\right)\left[\left(f_{1}+f_{2}\right) \gamma_{\mu}+\frac{\left(p_{1}+p_{2}\right)_{\mu}}{2 m_{B}} f_{2}\right] u_{B}\left(p_{2}\right) \varepsilon^{\mu}
\end{aligned}
$$


where the form factors $f_{i}$ are in general functions of $q^{2}=\left(p_{2}-p_{1}\right)^{2}$, and $\varepsilon^{\mu}$ is the polarization vector of the photon. In the problem under consideration, only the values of the form factors at $q^{2}=0$ are needed.

Using Eqs. (3)-(5), for the phenomenological part of the correlator (11) we obtain

$$
\Pi=-\lambda_{B}^{2} \varepsilon^{\mu} \frac{\not p_{1}+m_{B}}{p_{1}^{2}-m_{B}^{2}}\left[\left(f_{1}+f_{2}\right) \gamma_{\mu}+\frac{\left(p_{1}+p_{2}\right)_{\mu}}{2 m_{B}} f_{2}\right] \frac{\not p_{2}+m_{B}}{p_{2}^{2}-m_{B}^{2}}+\cdots,
$$

where $\cdots$ represents contributions from the higher states and the continuum. As is obvious from Eq. (6), the correlator function contains some number of different structures. Among all possible structures, we choose the one $\sim \not p_{1} \notin \not q$ that contains the magnetic form factor $f_{1}+f_{2}$. The value of $f_{1}+f_{2}$ at $q^{2}=0$ gives the magnetic moment of the baryon in units of its natural magneton, i.e., $e \hbar / 2 m_{B} c$.

Isolating the structure $\sim \not p_{1} \not \subset \not$ from the phenomenological part of the correlator, which describes the magnetic form factor, we get

$$
\Pi=-\lambda_{B}^{2} \frac{1}{p_{1}^{2}-m_{B}^{2}} \mu \frac{1}{p_{2}^{2}-m_{B}^{2}}+\cdots,
$$

where $\mu=\left.\left(f_{1}+f_{2}\right)\right|_{q^{2}=0}$.

Using the explicit form of the interpolating currents given in Eq. (2) and after some calculations, for the QCD part of the correlator functions of the $\Lambda$ and $\Sigma^{+}$baryons, we get

$$
\begin{aligned}
& \Pi^{\Lambda}=-\frac{2}{3} \epsilon_{a b c} \epsilon_{\text {def }} \int d^{4} x e^{i p x}\langle\gamma| \sum_{\ell=1}^{2} \sum_{k=1}^{2}\left\{4 A_{2}^{\ell} S_{s}^{b e}(x) A_{2}^{k} \operatorname{Tr} S_{d}^{c f}(x) C A_{1}^{k} S_{u}^{a d T}(x) C A_{1}^{\ell}\right. \\
& \quad+2 A_{2}^{\ell} S_{s}^{b e}(x) C A_{1}^{k} S_{u}^{a d T}(x) C A_{1}^{\ell} S_{d}^{c f}(x) A_{2}^{k}-2 A_{2}^{\ell} S_{s}^{b e}(x) C A_{1}^{k} S_{d}^{c f T}(x)\left(C A_{1}^{\ell}\right)^{T} S_{u}^{a d}(x) A_{2}^{k} \\
& \quad+2 A_{2}^{\ell} S_{d}^{c f}(x) C A_{1}^{k} S_{u}^{a d T}(x) C A_{1}^{\ell} S_{s}^{b e}(x) A_{2}^{k}+A_{2}^{\ell} S_{d}^{c f}(x) A_{2}^{k} \operatorname{Tr} S_{s}^{b e}(x) C A_{1}^{k} S_{u}^{a d T}(x) C A_{1}^{\ell} \\
& \quad-A_{2}^{\ell} S_{d}^{c f}(x)\left(C A_{1}^{k}\right)^{T} S_{s}^{b e T}(x)\left(C A_{1}^{\ell}\right)^{T} S_{u}^{a d}(x) A_{2}^{k}-2 A_{2}^{\ell} S_{u}^{a d}(x)\left(C A_{1}^{k}\right)^{T} S_{d}^{c f T}(x) C A_{1}^{\ell} S_{s}^{b e}(x) A_{2}^{k} \\
& \left.\quad-A_{2}^{\ell} S_{u}^{a d}(x)\left(C A_{1}^{k}\right)^{T} S_{s}^{b e T}(x)\left(C A_{1}^{\ell}\right)^{T} S_{d}^{c f}(x) A_{2}^{k}+A_{2}^{\ell} S_{u}^{a d}(x) A_{2}^{k} \operatorname{Tr}_{s}^{b e}(x) C A_{1}^{k} S_{d}^{c f T}(x) C A_{1}^{\ell}\right\}|0\rangle, \\
& \Pi^{\Sigma^{+}}=-2 \epsilon_{a b c} \epsilon_{d e f} \int d^{4} x e^{i p x}\langle\gamma| \sum_{\ell=1}^{2} \sum_{k=1}^{2}\left\{A_{2}^{\ell} S_{u}^{c f}(x) A_{2}^{k} \operatorname{Tr} S_{s}^{b e}(x) C A_{1}^{k} S_{u}^{a d T}(x) C A_{1}^{\ell}\right. \\
& \quad+A_{2}^{\ell}\left[S_{u}^{c f}(x)\left(C A_{1}^{k}\right)^{T} S_{s}^{b e T}(x)\left(C A_{1}^{\ell}\right)^{T} S_{u}^{a d}(x) A_{2}^{k}+S_{u}^{a d}(x)\left(C A_{1}^{k}\right)^{T} S_{s}^{b e T}(x)\left(C A_{1}^{\ell}\right)^{T} S_{u}^{c f}(x) A_{2}^{k}\right. \\
& \left.\left.\quad+S_{u}^{a d}(x) A_{2}^{k} \operatorname{Tr} S_{s}^{b e}(x) C A_{1}^{k} S_{d}^{c f T}(x) C A_{1}^{\ell}\right]\right\}|0\rangle,
\end{aligned}
$$

where $C$ is the charge conjugation operator, subscripts $a, b, c, d, e, f$ are the color indices and $S_{q}$ is the full propagator of the light quark involving both perturbative and nonperturbative parts. The expression for the theoretical parts of the correlator functions of the $\Sigma^{-}, \Sigma^{0}, \Xi^{0}$ and $\Xi^{-}$baryons can be obtained from Eq. (9) by the following replacements:

$$
\begin{aligned}
\Pi^{\Sigma^{-}} & =\Pi^{\Sigma^{+}}(u \rightarrow d), \\
\Pi^{\Xi^{0}} & =\Pi^{\Sigma^{+}}(u \leftrightarrow s), \\
\Pi^{\Xi^{-}} & =\Pi^{\Sigma^{+}}(u \rightarrow s, s \rightarrow d), \\
\Pi^{\Sigma^{0}} & =\frac{1}{2}\left(\Pi^{\Sigma^{-}}+\Pi^{\Sigma^{+}}\right), \\
\Pi^{p} & =\Pi^{\Xi^{-}}(s \rightarrow u) .
\end{aligned}
$$


Our calculations show that contributions from quadratic terms in strange quark mass $m_{s}$ are negligibly small compared to that of linear terms in $m_{s}$, which is about $6 \%$. Because of this reason theoretical part of the correlator function is calculated up to linear order in $m_{s}$. For the full light quark propagator we have used the following expression

$$
\begin{aligned}
& S_{q}(x)=\langle 0|T\{\bar{q}(x) q(0)\}| 0\rangle \\
& \quad=\frac{i \not x}{2 \pi^{2} x^{4}}-\frac{m_{q}}{4 \pi^{2} x^{2}}-\frac{\langle\bar{q} q\rangle}{12}\left(1-\frac{i m_{q}}{4} \not x\right)-\frac{x^{2}}{192} m_{0}^{2}\langle\bar{q} q\rangle\left(1-\frac{i m_{q}}{6} \not x\right) \\
& -i g_{s} \int_{0}^{1} d v\left[\frac{\not x}{16 \pi^{2} x^{2}} G_{\mu \nu}(v x) \sigma^{\mu \nu}-v x^{\mu} G_{\mu \nu}(v x) \gamma^{\nu} \frac{i}{4 \pi^{2} x^{2}}-\frac{i m_{q}}{32 \pi^{2}} G_{\mu \nu} \sigma^{\mu \nu}\left(\ln \frac{-x^{2} \Lambda^{2}}{4}+2 \gamma_{E}\right)\right],
\end{aligned}
$$

where $\Lambda$ is the energy cut off separating perturbative and nonperturbative regimes.

Few words about this expression of the light quark propagator are in order. The complete light cone expansion of the light quark propagator in external field has been carried in [17, and it has been shown that it gets contributions from nonlocal quark operators $\bar{q} G q, \bar{q} G G q, \bar{q} q \bar{q} q$, where $G_{\mu \nu}$ is the gluon field strength tensor. In the present work we consider the operators with only one-gluon field and neglect components with two-gluon and four-quark field. Formally, neglecting two-gluon and four-quark field terms can be justified on the basis of an expansion in conformal spin [18].

Perturbative part (i.e., photon interacting with quarks perturbatively) of the correlator function can be obtained by making the following substitution in one of the propagators in Eq. (9)

$$
S_{q_{\alpha \beta}}^{a b} \rightarrow-\frac{1}{2} e e_{q}\left(\int d y \mathcal{F}^{\mu \nu} y_{\nu} S_{q}^{f r e e}(x-y) \gamma_{\mu} S_{q}^{f r e e}(y)\right)_{\alpha \beta}^{a b}
$$

where the Fock-Schwinger gauge $x^{\mu} A_{\mu}(x)=0$ is used and $S_{q}^{\text {free }}=i \not x /\left(2 \pi^{2} x^{4}\right)$ is the free propagator, the remaining two propagators are the full propagators of the quarks (see Eq. (9)) and $\mathcal{F}^{\mu \nu}$ is the electromagnetic field strength tensor. The explicit expression of the nonperturbative contribution can be obtained from Eq. (8) by replacing one of the propagators

$$
S_{q_{\alpha \beta}}^{a b} \rightarrow-\frac{1}{4} \bar{q}^{a} A_{j} q^{b}\left(A_{j}\right)_{\alpha \beta}
$$

where $A_{j}=\left\{1, \gamma_{5}, \gamma_{\alpha}, i \gamma_{5} \gamma_{\alpha}, \sigma_{\alpha \beta} / \sqrt{2}\right\}$ is the full set of Dirac matrices and sum over $A_{j}$ is implied, and the other two propagators are the full ones.

It follows from Eqs. (8) and (9) that in order to calculate the nonperturbative contributions to the theoretical part of the correlator functions, one needs to know the matrix elements $\left\langle\gamma\left|\bar{q} A_{j} q\right| 0\right\rangle$ of the nonlocal operators between photon and vacuum states. Up to twist-4, non-zero matrix elements of the nonlocal operators are determined in terms of the photon wave functions as follows [19, 20]:

$$
\begin{aligned}
\left\langle\gamma(q)\left|\bar{q} \gamma_{\alpha} \gamma_{5} q\right| 0\right\rangle & =\frac{f}{4} e_{q} \epsilon_{\alpha \beta \rho \sigma} \varepsilon^{\beta} q^{\rho} x^{\sigma} \int_{0}^{1} d u e^{i u q x} \psi(u) \\
\left\langle\gamma(q)\left|\bar{q} \sigma_{\alpha \beta} q\right| 0\right\rangle & =i e_{q}\langle\bar{q} q\rangle \int_{0}^{1} d u e^{i u q x}\left\{\left(\varepsilon_{\alpha} q_{\beta}-\varepsilon_{\beta} q_{\alpha}\right)\left[\chi \phi(u)+x^{2}\left(g_{1}(u)-g_{2}(u)\right)\right]\right. \\
& \left.+\left[q x\left(\varepsilon_{\alpha} x_{\beta}-\varepsilon_{\beta} x_{\alpha}\right)+\varepsilon x\left(x_{\alpha} q_{\beta}-x_{\beta} q_{\alpha}\right)\right] g_{2}(u)\right\}
\end{aligned}
$$


where $\chi$ is the magnetic susceptibility of the quark condensate, $e_{q}$ is the quark charge, $\phi(u)$ and $\psi(u)$ are the leading twist -2 photon wave functions, while $g_{1}(u)$ and $g_{2}(u)$ are the twist -4 functions, respectively. Note that twist -3 photon wave functions are neglected in further calculations since their contribution changes the results about $5 \%$.

Using Eqs. (11) and (14), we can calculate theoretical part of the correlator functions for $\Lambda$ and $\Sigma^{+}$baryons from Eqs. (8) and (9). The sum rule is obtained by equating the theoretical and phenomenological parts of the corresponding correlator function. Performing double Borel transformations on the variables $p_{1}^{2}=p^{2}$ and $p_{2}^{2}=(p+q)^{2}$ in order to suppress the continuum and higher state contributions (for a discussion concerning this point, see [21]-23]), the following sum rules for the $\Lambda$ and $\Sigma^{+}$baryons are obtained:

$$
\begin{aligned}
& \lambda_{\Lambda}^{2} \mu_{\Lambda} e^{-M^{2} / M_{\Lambda}^{2}}= \\
& \frac{1}{192 \pi^{4}} M^{6} E_{2}(x)\left[\left(-1+t^{2}\right)\left(e_{u}+e_{d}\right)+\left(13+10 t+13 t^{2}\right) e_{s}\right] \\
& -\frac{m_{s}}{48 \pi^{2}} M^{4} E_{1}(x)\left(-5+4 t+t^{2}\right) \chi \varphi\left(u_{0}\right)\langle\bar{q} q\rangle\left(e_{u}+e_{d}\right) \\
& -\frac{1}{96 \pi^{2}} M^{4} E_{1}(x) f \psi\left(u_{0}\right)\left[(-1+t)^{2}\left(e_{u}+e_{d}\right)+\left(13+10 t+13 t^{2}\right) e_{s}\right] \\
& +\frac{m_{s}}{12 \pi^{2}} M^{2} E_{0}(x)\left(-1-4 t+5 t^{2}\right)\langle\bar{q} q\rangle\left(\gamma_{E}-\ln \frac{M^{2}}{\Lambda^{2}}\right) e_{s} \\
& -\frac{m_{s}}{288 \pi^{2}} m_{0}^{2}\langle\bar{q} q\rangle\left[9\left(-1+t^{2}\right)\left(e_{u}+e_{d}\right)+\left(-3-12 t+16 t^{2}\right) e_{s}\right]\left(\gamma_{E}-\ln \frac{M^{2}}{\Lambda^{2}}\right) \\
& -\frac{2}{9}\left[g_{1}\left(u_{0}\right)-g_{2}\left(u_{0}\right)\right]\langle\bar{q} q\rangle\left[\left(-5+4 t+t^{2}\right)\langle\bar{s} s\rangle+(-1+t)^{2}\langle\bar{q} q\rangle\right]\left(e_{u}+e_{d}\right) \\
& +\frac{m_{s}}{6 \pi^{2}}\left(-5+4 t+t^{2}\right)\left[g_{1}\left(u_{0}\right)-g_{2}\left(u_{0}\right)\right] M^{2} E_{0}(x)\langle\bar{q} q\rangle\left(e_{u}+e_{d}\right) \\
& +\frac{4}{9}\left(-1-4 t-5 t^{2}\right)\left[g_{1}\left(u_{0}\right)-g_{2}\left(u_{0}\right)\right]\langle\bar{s} s\rangle\langle\bar{u} u\rangle e_{s} \\
& +\frac{m_{s}}{48 \pi^{2}} M^{2} E_{0}(x)\left[(-1+t)^{2}\langle\bar{s} s\rangle-2\left(-5+4 t+t^{2}\right)\langle\bar{u} u\rangle\right]\left(e_{u}+e_{d}\right) \\
& +\frac{m_{s}}{12 \pi^{2}} M^{2}\langle\bar{u} u\rangle\left(-1-4 t+5 t^{2}\right) e_{s} \\
& +\frac{1}{18} M^{2} E_{0}(x) \chi \varphi\left(u_{0}\right)\langle\bar{q} q\rangle\left[\left(-5+4 t+t^{2}\right)\langle\bar{s} s\rangle+(-1+t)^{2}\langle\bar{q} q\rangle\right]\left(e_{u}+e_{d}\right) \\
& +\frac{1}{9} M^{2} E_{0}(x)\left(1+4 t-5 t^{2}\right) \chi \varphi\left(u_{0}\right)\langle\bar{s} s\rangle\langle\bar{q} q\rangle e_{s} \\
& -\frac{m_{s}}{72} f \psi\left(u_{0}\right)\left[(-1+t)^{2}\langle\bar{s} s\rangle-2\left(-5+4 t+t^{2}\right)\langle\bar{q} q\rangle\right]\left(e_{u}+e_{d}\right) \\
& -\frac{1}{144} m_{0}^{2} \chi \varphi\left(u_{0}\right)\langle\bar{u} u\rangle\left[2\left(-5+4 t+t^{2}\right)\langle\bar{s} s\rangle+\left(-3-2 t+5 t^{2}\right)\langle\bar{q} q\rangle\right]\left(e_{u}+e_{d}\right) \\
& +\frac{1}{216} m_{0}^{2}\left(-3-12 t+16 t^{2}\right) \chi \varphi\left(u_{0}\right)\langle\bar{s} s\rangle\langle\bar{q} q\rangle e_{s} \\
& +\frac{1}{18}\langle\bar{q} q\rangle\left[\left(-5+4 t+t^{2}\right)\langle\bar{s} s\rangle\left(e_{u}+e_{d}\right)+\left(-11-2 t+13 t^{2}\right)\langle\bar{q} q\rangle e_{d}\right] \\
& -\frac{m_{s}}{288 \pi^{2}} m_{0}^{2}\left[(-1+t)^{2}\langle\bar{s} s\rangle-3\left(-5+4 t+t^{2}\right)\langle\bar{q} q\rangle\right]\left(e_{u}+e_{d}\right) \text {, }
\end{aligned}
$$




$$
\begin{aligned}
& \lambda_{\Sigma^{+}}^{2} \mu_{\Sigma^{+}} e^{-M^{2} / M_{\Sigma^{+}}^{2}}= \\
& -\frac{1}{64 \pi^{4}}\left[M^{6} E_{2}(x)-2 M^{4} E_{1}(x) f \psi\left(u_{0}\right)\right]\left[(1+t)^{2} e_{s}-2\left(3+2 t+3 t^{2}\right) e_{u}\right] \\
& +\frac{m_{u}}{4 \pi^{2}} M^{2} E_{0}(x)\left[\left(-1+t^{2}\right)\langle\bar{s} s\rangle+(-1+t)^{2}\langle\bar{u} u\rangle\right] e_{u}\left(\gamma_{E}-\ln \frac{M^{2}}{\Lambda^{2}}\right) \\
& -\frac{m_{s}}{4 \pi^{2}} M^{2} E_{0}(x)\left(-1+t^{2}\right)\langle\bar{u} u\rangle e_{s}\left(\gamma_{E}-\ln \frac{M^{2}}{\Lambda^{2}}\right) \\
& +\frac{m_{s}}{192 \pi^{2}} m_{0}^{2}\langle\bar{u} u\rangle\left[\left(-15+14 t^{2}\right) e_{s}-12\left(-3+t+3 t^{2}\right) e_{u}\right]\left(\gamma_{E}-\ln \frac{M^{2}}{\Lambda^{2}}\right) \\
& -\frac{m_{u}}{96 \pi^{2}} m_{0}^{2}\left[\left(-7+19 t^{2}\right)\langle\bar{s} s\rangle+3\left(1-3 t+t^{2}\right)\langle\bar{u} u\rangle\right] e_{u}\left(\gamma_{E}-\ln \frac{M^{2}}{\Lambda^{2}}\right) \\
& -\frac{4}{3}\left(-1+t^{2}\right)\left[g_{1}\left(u_{0}\right)-g_{2}\left(u_{0}\right)\right]\langle\bar{s} s\rangle\langle\bar{u} u\rangle e_{s} \\
& +\frac{4}{3}\left[g_{1}\left(u_{0}\right)-g_{2}\left(u_{0}\right)\right]\langle\bar{u} u\rangle\left[\left(-1+t^{2}\right)\langle\bar{s} s\rangle+(-1+t)^{2}\langle\bar{u} u\rangle\right] e_{u} \\
& -\frac{1}{\pi^{2}}\langle\bar{u} u\rangle\left[g_{1}\left(u_{0}\right)-g_{2}\left(u_{0}\right)\right] M^{2} E_{0}(x)\left[\left(-1+t^{2}\right) m_{s}+(-1+t)^{2} m_{u}\right] e_{u} \\
& +\frac{1}{\pi^{2}}\left(-1+t^{2}\right)\left[g_{1}\left(u_{0}\right)-g_{2}\left(u_{0}\right)\right] M^{2} E_{0}(x) m_{u}\langle\bar{s} s\rangle e_{s} \\
& +\frac{1}{8 \pi^{2}} M^{4} E_{1}(x)\left[\left(-1+t^{2}\right) m_{s}+(-1+t)^{2} m_{u}\right] \chi \varphi\left(u_{0}\right)\langle\bar{u} u\rangle e_{u} \\
& -\frac{m_{u}}{8 \pi^{2}} M^{4} E_{1}(x)\left(-1+t^{2}\right) \chi \varphi\left(u_{0}\right)\langle\bar{s} s\rangle e_{s} \\
& +\frac{1}{3}\left(-1+t^{2}\right) M^{2} E_{0}(x) \chi \varphi\left(u_{0}\right)\langle\bar{s} s\rangle\langle\bar{u} u\rangle e_{s} \\
& -\frac{1}{3} M^{2} E_{0}(x) \chi \varphi\left(u_{0}\right)\langle\bar{u} u\rangle\left[\left(-1+t^{2}\right)\langle\bar{s} s\rangle+(-1+t)^{2}\langle\bar{u} u\rangle\right] e_{u} \\
& +\frac{m_{u}}{8 \pi^{2}} M^{2} E_{0}(x)\left[-4\left(-1+t^{2}\right)\langle\bar{s} s\rangle+\left(5-2 t+5 t^{2}\right)\langle\bar{u} u\rangle\right] e_{u} \\
& +\frac{m_{u}}{8 \pi^{2}} M^{2} E_{0}(x)\left(1-6 t+t^{2}\right)\langle\bar{u} u\rangle e_{s} \\
& +\frac{m_{s}}{8 \pi^{2}} M^{2} E_{0}(x)\left[\left(3+2 t+3 t^{2}\right)\langle\bar{s} s\rangle-6\left(-1+t^{2}\right)\langle\bar{u} u\rangle\right] e_{u} \\
& -\frac{m_{s}}{4 \pi^{2}} M^{2} E_{0}(x)\left(-1+t^{2}\right)\langle\bar{u} u\rangle e_{s} \\
& +\frac{m_{u}}{12} f \psi\left(u_{0}\right)\left[6\left(-1+t^{2}\right)\langle\bar{s} s\rangle-\left(3+2 t+3 t^{2}\right)\langle\bar{u} u\rangle\right] e_{u} \\
& -\frac{m_{u}}{12} f \psi\left(u_{0}\right)\left(1-6 t+t^{2}\right)\langle\bar{u} u\rangle e_{s} \\
& -\frac{m_{s}}{12} f \psi\left(u_{0}\right)\left[\left(3+2 t+3 t^{2}\right)\langle\bar{s} s\rangle-6\left(-1+t^{2}\right)\langle\bar{u} u\rangle\right] e_{u} \\
& -\frac{1}{6}\langle\bar{u} u\rangle\left[(-1+t)^{2}\langle\bar{u} u\rangle e_{s}-6\left(-1+t^{2}\right)\langle\bar{s} s\rangle e_{u}\right] \\
& +\frac{1}{72} m_{0}^{2} \chi \varphi\left(u_{0}\right)\langle\bar{u} u\rangle\left[\left(-1+t^{2}\right)\langle\bar{s} s\rangle+3\left(1-3 t+t^{2}\right)\langle\bar{u} u\rangle\right] e_{u}
\end{aligned}
$$




$$
\begin{aligned}
& +\frac{1}{144} m_{0}^{2}\left(15-14 t^{2}\right) \chi \varphi\left(u_{0}\right)\langle\bar{s} s\rangle\langle\bar{u} u\rangle e_{s} \\
& +\frac{m_{u}}{48 \pi^{2}} m_{0}^{2}\left[9\left(-1+t^{2}\right)\langle\bar{s} s\rangle-\left(3+2 t+3 t^{2}\right)\langle\bar{u} u\rangle\right] e_{u} \\
& -\frac{m_{u}}{24 \pi^{2}} m_{0}^{2}\left(1-4 t+t^{2}\right)\langle\bar{u} u\rangle e_{s} \\
& -\frac{m_{s}}{48 \pi^{2}} m_{0}^{2}\left[\left(3+2 t+3 t^{2}\right)\langle\bar{s} s\rangle-9\left(-1+t^{2}\right)\langle\bar{u} u\rangle\right] e_{u} .
\end{aligned}
$$

In Eqs. (15) and (16) the functions

$$
E_{n}(x)=1-e^{x} \sum_{k=0}^{n} \frac{x^{k}}{k},
$$

are used to subtract the continuum and higher state contributions, $x=s_{0} / M^{2}$ and $s_{0}$ is the continuum threshold. Moreover

$$
\begin{aligned}
u_{0} & =\frac{M_{2}^{2}}{M_{1}^{2}+M_{2}^{2}}, \\
M^{2} & =\frac{M_{1}^{2} M_{2}^{2}}{M_{1}^{2}+M_{2}^{2}},
\end{aligned}
$$

but, since we are dealing with just a single baryon, the Borel parameters $M_{1}^{2}$ and $M_{2}^{2}$ should be set to equal each other, from which it follows that $u_{0}=1 / 2$.

As has already been noted, the sum rules for the $\Sigma^{0}, \Sigma^{-}, \Xi^{0}, \Xi^{-}, p, n$ baryons can be obtained by making the following replacements:

$$
\begin{aligned}
\mu_{\Sigma^{0}} & =\mu_{\Sigma^{+}}\left(m_{\Sigma^{+}} \rightarrow m_{\Sigma^{0}}, \lambda_{\Sigma^{+}} \rightarrow \lambda_{\Sigma^{0}}, e_{u} \rightarrow \frac{e_{u}+e_{d}}{2}, s_{0}^{\Sigma^{+}} \rightarrow s_{0}^{\Sigma^{0}}\right), \\
\mu_{\Sigma^{-}} & =\mu_{\Sigma^{+}}\left(m_{\Sigma^{+}} \rightarrow m_{\Sigma^{-}}, \lambda_{\Sigma^{+}} \rightarrow \lambda_{\Sigma^{-}}, e_{u} \rightarrow e_{d}, s_{0}^{\Sigma^{+}} \rightarrow s_{0}^{\Sigma^{-}}\right), \\
\mu_{\Xi^{0}} & =\mu_{\Sigma^{+}}\left(m_{\Sigma^{+}} \rightarrow m_{\Xi^{0}}, e_{s} \leftrightarrow e_{u}, m_{s} \leftrightarrow m_{u},\langle\bar{s} s\rangle \rightarrow\langle\bar{u} u\rangle, s_{0}^{\Sigma^{+}} \rightarrow s_{0}^{\Xi^{0}}\right), \\
\mu_{\Xi^{-}} & =\mu_{\Xi^{0}}\left(m_{\Xi^{0}} \rightarrow m_{\Xi^{-}}, e_{u} \leftrightarrow e_{d}, s_{0}^{\Xi^{0}} \leftrightarrow s_{0}^{\Xi^{-}}\right), \\
\mu_{p} & =\mu_{\Xi^{-}}\left(m_{\Xi^{-}} \rightarrow m_{p}, e_{s} \rightarrow e_{u}, m_{s} \rightarrow m_{u},\langle\bar{s} s\rangle \rightarrow\langle\bar{u} u\rangle, s_{0}^{\Xi^{-}} \rightarrow s_{0}^{p}\right), \\
\mu_{n} & =\mu_{p}\left(e_{u} \rightarrow \frac{e_{u}+e_{d}}{2}, s_{0}^{p} \rightarrow s_{0}^{n}\right) .
\end{aligned}
$$

Note that, after we make these replacements we set masses of $u$ and $d$ quarks to zero and assume $S U(2)$ flavor symmetry, which implies that $\langle\bar{u} u\rangle=\langle\bar{d} d\rangle$.

Obviously, it follows from Eqs. (15) and (16) that one needs to know the residues $\lambda_{B}$ of the octet baryons in order to determine their magnetic moments. These residues are determined from mass sum rules of the corresponding baryons:

$$
\begin{aligned}
& \lambda_{\Sigma^{+}}^{2} e^{-m_{\Sigma^{+}}^{2} / M^{2}}= \\
& \quad \frac{M^{6}}{256 \pi^{4}} E_{2}(x)\left(5+2 t+5 t^{2}\right)-\frac{m_{0}^{2}}{24 M^{2}}\langle\bar{u} u\rangle\left[12\left(-1+t^{2}\right)\langle\bar{s} s\rangle+(-1+t)^{2}\langle\bar{u} u\rangle\right] \\
& +\frac{m_{s}}{32 \pi^{2}} M^{2} E_{0}(x)\left[\left(5+2 t+5 t^{2}\right)\langle\bar{s} s\rangle-12\left(-1+t^{2}\right)\langle\bar{u} u\rangle\right]
\end{aligned}
$$




$$
\begin{aligned}
& -\frac{m_{0}^{2}}{96 \pi^{2}} m_{s}\left[\left(5+2 t+5 t^{2}\right)\langle\bar{s} s\rangle-3\left(-1+t^{2}\right)\langle\bar{u} u\rangle\right] \\
& +\frac{\langle\bar{u} u\rangle}{6}\left[6\left(-1+t^{2}\right)\langle\bar{s} s\rangle+(-1+t)^{2}\langle\bar{u} u\rangle\right] \\
& -\frac{3 m_{s}}{16 \pi^{2}} m_{0}^{2}\langle\bar{u} u\rangle\left(-1+t^{2}\right)\left(\gamma_{E}-\ln \frac{M^{2}}{\Lambda^{2}}\right) \\
& \lambda_{\Xi^{-}}^{2} e^{-m_{\Xi^{-}}^{2} / M^{2}}= \\
& \frac{M^{6}}{256 \pi^{4}} E_{2}(x)\left(5+2 t+5 t^{2}\right)-\frac{m_{0}^{2}}{24 M^{2}}\langle\bar{s} s\rangle\left[12\left(-1+t^{2}\right)\langle\bar{d} d\rangle+(-1+t)^{2}\langle\bar{s} s\rangle\right] \\
& +\frac{3 m_{s}}{16 \pi^{2}} M^{2} E_{0}(x)\left[-2\left(-1+t^{2}\right)\langle\bar{d} d\rangle+(1+t)^{2}\langle\bar{s} s\rangle\right] \\
& +\frac{\langle\bar{s} s\rangle}{6}\left[6\left(-1+t^{2}\right)\langle\bar{d} d\rangle+(-1+t)^{2}\langle\bar{s} s\rangle\right] \\
& -\frac{m_{0}^{2} m_{s}}{96 \pi^{2}}\left[3\left(-1+t^{2}\right)\langle\bar{d} d\rangle+\left(7+10 t+7 t^{2}\right)\langle\bar{s} s\rangle\right] \\
& -\frac{3 m_{0}^{2} m_{s}}{16 \pi^{2}}\langle\bar{d} d\rangle\left(-1+t^{2}\right)\left(\gamma_{E}-\ln \frac{M^{2}}{\Lambda^{2}}\right) \\
& \lambda_{\Lambda}^{2} e^{-M_{\Lambda}^{2} / M^{2}}= \\
& \frac{1}{256 \pi^{4}}\left(5+2 t+5 t^{2}\right) M^{6} E_{2}(x) \\
& +\frac{1}{72}(1-t) \frac{m_{0}^{2}}{M^{2}}\{4(1+2 t)\langle\bar{s} s\rangle(\langle\bar{u} u\rangle+\langle\bar{d} d\rangle)+(25+23 t)\langle\bar{u} u\rangle\langle\bar{d} d\rangle\} \\
& +\frac{m_{s}}{96 \pi^{2}} M^{2} E_{0}(x)\left\{3\left(5+2 t+5 t^{2}\right)\langle\bar{s} s\rangle+2\left(1+4 t-5 t^{2}\right)(\langle\bar{u} u\rangle+\langle\bar{d} d\rangle)\right\} \\
& +\frac{m_{s}}{32 \pi^{2}} m_{0}^{2}(\langle\bar{u} u\rangle+\langle\bar{d} d\rangle)\left(1-t^{2}\right)\left\{\gamma_{E}-\ln \left(\frac{M^{2}}{\Lambda^{2}}\right)\right\} \\
& -\frac{1}{18}(1-t)\{(1+5 t)\langle\bar{s} s\rangle(\langle\bar{u} u\rangle+\langle\bar{d} d\rangle)+(13+11 t)\langle\bar{u} u\rangle\langle\bar{d} d\rangle\} \\
& -\frac{m_{s}}{192 \pi^{2}} m_{0}^{2}\left\{2\left(5+2 t+5 t^{2}\right)\langle\bar{s} s\rangle+\left(-5+4 t+t^{2}\right)(\langle\bar{u} u\rangle+\langle\bar{d} d\rangle)\right\} \\
& \lambda_{\Sigma^{0}}^{2} e^{-M_{\Sigma^{0}}^{2} / M^{2}}= \\
& \frac{1}{256 \pi^{4}}\left(5+2 t+5 t^{2}\right) M^{6} E_{2}(x) \\
& +\frac{m_{s}}{32 \pi^{2}} M^{2} E_{0}(x)\left\{\left(5+2 t+5 t^{2}\right)\langle\bar{s} s\rangle-6\left(-1+t^{2}\right)(\langle\bar{u} u\rangle+\langle\bar{d} d\rangle)\right\} \\
& +\frac{1}{24} \frac{m_{0}^{2}}{M^{2}}(1-t)\{6(1+t)\langle\bar{s} s\rangle(\langle\bar{u} u\rangle+\langle\bar{d} d\rangle)+(-1+t)\langle\bar{u} u\rangle\langle\bar{d} d\rangle\} \\
& +\frac{3 m_{s}}{32 \pi^{2}} m_{0}^{2}(\langle\bar{u} u\rangle+\langle\bar{d} d\rangle)\left(1-t^{2}\right)\left\{\gamma_{E}-\ln \left(\frac{M^{2}}{\Lambda^{2}}\right)\right\} \\
& -\frac{m_{s}}{192 \pi^{2}} m_{0}^{2}\left\{2\left(5+2 t+5 t^{2}\right)\langle\bar{s} s\rangle-3\left(-1+t^{2}\right)(\langle\bar{u} u\rangle+\langle\bar{d} d\rangle)\right\}
\end{aligned}
$$




$$
-\frac{1}{6}(1-t)\{3(1+t)\langle\bar{s} s\rangle(\langle\bar{u} u\rangle+\langle\bar{d} d\rangle)+(-1+t)\langle\bar{u} u\rangle\langle\bar{d} d\rangle\}
$$

\section{Numerical Analysis}

In this section we present the numerical analysis of the sum rules for the magnetic moments of the octet baryons. The main input parameters in the LCQCD are the photon wave functions which have been introduced in the previous section. In [18] and 19] it has been shown that the leading photon wave functions do not deviate much from their asymptotic form. Therefore in numerical calculations we shall use the following forms of the photon wave functions [18, 20]

$$
\begin{aligned}
\phi(u) & =6 u(1-u), \quad \psi(u)=1, \\
g_{1}(u) & =-\frac{1}{8}(1-u)(3-u), \quad g_{2}(u)=-\frac{1}{4}(1-u)^{2} .
\end{aligned}
$$

The values of the other input parameters that are used in the numerical analysis are: $f=$ $0.028 \mathrm{GeV}^{2}, \chi=-3.3 \mathrm{GeV}^{-2}$ [24] (in [25] this quantity is estimated to be $\chi=-4.4 \mathrm{GeV}^{-2}$ ), $\langle\bar{q} q\rangle(1 \mathrm{GeV})=-(0.243)^{3} \mathrm{GeV}^{3}, m_{0}^{2}=(0.8 \pm 0.2) \mathrm{GeV}^{2}$ [26], $m_{s}(1 \mathrm{GeV})=(150 \pm 50) \mathrm{MeV}$, $\langle\bar{s} s\rangle(1 \mathrm{GeV})=0.8\langle\bar{q} q\rangle(1 \mathrm{GeV})$ and $\Lambda=0.5 \mathrm{GeV}$. In the problem under consideration we have three auxiliary parameters, namely parameter $t$ in the hadron interpolating currents, Borel mass square $M^{2}$ and the continuum threshold $s_{0}$. No doubt, it is expected that any physically measurable quantity must be independent of these auxiliary parameters. So, our problem is to find the appropriate regions, for which magnetic moments of octet baryons are independent of the above-mentioned parameters.

For this aim we consider the following three-step procedure. In the first step we attempt to find working region of $M^{2}$, where magnetic moments of octet baryons are independent of the Borel parameter at fixed values of $s_{0}$ and $t$. In Figs. (1)-(8) we present the dependence of the magnetic moments of the corresponding baryons on $M^{2}$. From these figures we see that the magnetic moments of octet baryons seem to be almost independent of $M^{2}$ for different choices of $t$ and $s_{0}$. However, the working region for the Borel mass square parameter for the members of octet baryons are different, i.e., $0.9 \mathrm{GeV}^{2}<M^{2}<1.2 \mathrm{GeV}^{2}$ for $p, n ; 1.3 \mathrm{GeV}^{2}<M^{2}<1.6 \mathrm{GeV}^{2}$ for $\Sigma^{-}, \Sigma^{0}, \Sigma^{+}$and $\Lambda ; 1.7 \mathrm{GeV}^{2}<M^{2}<2.1 \mathrm{GeV}^{2}$ for $\Xi^{0}$ and $\Xi^{-}$.

Before determining the magnetic moment of baryons, the next problem to be considered is to find the working region of $t$, i.e. the region where the sum rules make sense. For this purpose we consider the mass sum rules (Eqs. (17 20)). Two criteria should be satisfied for mass sum rules. First of all, each mass sum rule must separately be positive. The second criteria is that, the predicted mass of the baryons which is obtained by taking the logarithmic derivative of the corresponding sum rule with respect to $M^{-2}$ should be stable with respect to the variation of the parameter $t$. As a result of analysis of the mass sum rules we found that, the region $-0.5<t<0.5$ is unphysical (see Fig. (9)). Our analysis shows that the working region of $t$ where the second criteria is satisfied is given by $-0.6<\cos \theta<0.3$, where $\theta$ is defined through the relation $\theta=\tan ^{-1} t$. This region of $\theta$ corresponds to $t<-1.4$ and $t>3.3$. We observe that the Ioffe current, which corresponds to the choice $t=-1$ does not lie in the working region. 
Our last attempt is to determine a region for the parameter $t$ where the magnetic moments of the octet baryons are independent of its variation. Here we stress again that in the first step the working region for the Borel mass square $M^{2}$ has been determined for which $\mu_{B}$ is practically independent of its variation, as well as insensitive to the continuum threshold. We present in Fig (10) the dependence of the magnetic moments of the octet baryons (in units of the nucleon magneton $\mu_{N}$ ) on $\cos \theta$, at fixed values of $M^{2}$ (remember that the fixed values of $M^{2}$ are different for each one of the octet baryons) and at fixed value of $s_{0}$ (again different fixed values of $s_{0}$ are used for each baryon). From this figure we observe that $\mu_{B}$ is practically independent of the parameter $t$ in the region $-0.6<\cos \theta<0.3$ and insensitive to the variation of the continuum threshold $s_{0}$. Our final results on the magnetic moments of the octet baryons are presented in Table (1) . For completeness, in this table we also present the predictions of other approaches on the magnetic moments of octet baryons and the experimental results as well as our results for the value of the magnetic susceptibility $\chi=-4.4 \mathrm{GeV}^{2}$. It follows from our analysis that light cone QCD sum rule prediction on magnetic moments of octet baryons are close to their experimental values for $\chi=-3.3 \mathrm{GeV}^{2}$, but considerable departure is observed for $\chi=-4.4 \mathrm{GeV}^{2}$. Obviously, it follows from Table 1 that similar situation takes place for the traditional sum rule predictions on the magnetic moments of octet baryons. The uncertainties in the results we have presented could be attributed to the variation of $s_{0}$, Borel parameter $M^{2}$, twist-3 photon wave functions which are neglected and errors in the input parameters such as $\langle\bar{q} q\rangle$, $m_{0}^{2}$ and $m_{s}$. All errors are added quadratically to the final predictions. It should be stressed here that the predictions on magnetic moments at $t=-1$, which corresponds to the Ioffe current, are not reliable.

From our results we see that the values of $\mu_{\Sigma^{+}} \approx \mu_{p} ; 2 \mu_{\Lambda} \approx \mu_{n} ; \mu_{\Xi^{0}} \approx \mu_{n}$ and $\mu_{\Xi^{-}} \approx \mu_{\Sigma^{-}}$. It should be added here that, in exact $S U(3)$ symmetry, the relations between the magnetic moments are as follows [27]:

$$
\begin{aligned}
\mu_{\Sigma^{+}} & =\mu_{p}, \\
2 \mu_{\Lambda} & =\mu_{n}, \\
\mu_{\Sigma^{-}}+\mu_{n} & =-\mu_{p}, \\
\mu_{\Xi^{-}} & =\mu_{\Sigma^{-}}, \\
\mu_{\Xi^{0}} & =\mu_{n} .
\end{aligned}
$$

Violation to the relations (21) comes from $S U(3)$ breaking terms (the mass of the $s$ quark, $s$ quark condensate, etc.). So, our predictions give us a clue about sizable $S U(3)$ symmetry breaking effects.

In summary, we have calculated magnetic moments of the octet baryons in light cone QCD sum rules, using the general form of the baryonic interpolating currents. We have obtained that light cone QCD sum rule predictions on magnetic moments of the octet baryons are in good agreement with the experimental data at $\chi=-3.3 \mathrm{GeV}^{2}$.

\section{Acknowledgements}

We would like to thank V. S. Zamiralov for fruitful discussions. 
Note added: After we have completed the present work, we have received a recent work [33] where the mass sum rules for general form of the interpolating currents for the baryons are calculated. Our results on mass sum rules coincide with with the ones predicted in this work. 


\begin{tabular}{|c|c|c|c|c|c|c|c|c|c|c|}
\hline & \multirow{2}{*}{ NQM } & \multirow{2}{*}{ SQM } & \multicolumn{2}{|c|}{ QCDSR } & \multirow{2}{*}{$\mathrm{QCDSA}$} & \multirow{2}{*}{$\chi \mathrm{PT}$} & \multirow{2}{*}{ SKRM } & \multicolumn{2}{|c|}{ LCQSR } & \multirow{2}{*}{ EXP } \\
\hline & & & $\chi=-3.3$ & $\chi=-4.4$ & & & & $\chi=-3.3$ & $\chi=-4.4$ & \\
\hline$\mu_{p}$ & 2.87 & 2.75 & 2.72 & 3.55 & 2.54 & 2.793 & 2.36 & $2.7 \pm 0.5$ & $3.5 \pm 0.5$ & 2.79 \\
\hline$\mu_{n}$ & -1.91 & -1.84 & -1.65 & -2.06 & -1.69 & -1.913 & -1.87 & $-1.8 \pm 0.35$ & $-2.3 \pm 0.4$ & -1.91 \\
\hline$\mu_{\Sigma^{+}}$ & 2.62 & 2.65 & 2.52 & 3.30 & 2.48 & 2.458 & 2.46 & $2.2 \pm 0.4$ & $2.9 \pm 0.4$ & $2.46 \pm 0.01$ \\
\hline$\mu_{\Sigma^{-}}$ & -1.20 & -1.02 & -1.13 & -1.38 & -0.90 & -1.16 & -1.16 & $-0.8 \pm 0.2$ & $-1.1 \pm 0.3$ & $-1.16 \pm 0.03$ \\
\hline$\mu_{\Xi^{0}}$ & -0.63 & -1.44 & -0.89 & -0.98 & -1.49 & -1.25 & -1.25 & $-1.3 \pm 0.3$ & $-1.3 \pm 0.4$ & $-1.25 \pm 0.01$ \\
\hline$\mu_{\Xi^{-}}$ & -1.44 & -0.52 & -1.18 & -1.27 & -0.63 & -0.6531 & -0.65 & $-0.7 \pm 0.2$ & $-1.0 \pm 0.2$ & -0.65 \\
\hline$\mu_{\Lambda}$ & -0.63 & -0.67 & -0.50 & -0.80 & -0.69 & -0.613 & -0.60 & $-0.7 \pm 0.2$ & $-0.9 \pm 0.2$ & -0.61 \\
\hline
\end{tabular}

Table 1: Predictions of various approaches for the octet baryon magnetic moments: naive quark model (NQM, see ref. in [31]); static quark model (SQM) [32]; QCD sum rules (QCDSR) [9]; QCD string approach (QCDSA) [28]; chiral perturbation theory $(\chi \mathrm{PT})[29]$; skyrme model (SKRM) [30]; present work (LCQSR). For completeness we present the experimental values of the octet baryons. All the values in the table are given in units of nuclear magneton $\mu_{N}$. 


\section{References}

[1] M. A. Shifman, V. I. Vainshtein, V. I. Zakharov, Nucl. Phys. B147 (1979) 385.

[2] L. J. Reinders, H. Rubinstein and S. Yazaki, Phys. Rept. 127 (1985) 1.

[3] Vacuum structure and QCD sum rules, Ed: M. A. Shifman, North-Holland, Amsterdam (1992).

[4] M. A. Shifman, Prog. Theor. Phys. Suppl. 131 (1998) 1.

[5] S. Narison, "QCD spectral sum rules", World Scientific (1989).

[6] P. Colangelo and A. Khodjamirian, "At the Frontier of Particle Physics / Handbook of QCD", 1495, ed. by M. Shifman (World Scientific, Singapore, 2001).

[7] B. L. Ioffe and A. V. Smilga, Nucl. Phys. B232 (1984) 109;

B. L. Ioffe and A. V. Smilga, Phys. Lett. B133 (1983) 436.

[8] I. I. Balitsky and A. V. Yung, Phys. Lett. B129 (1983) 328.

[9] C. B. Chiu, J. Pasupathy, S. L. Wilson, Phys. Rev. D33 (1986) 1961.

[10] J. Pasupathy, J. P. Singh, S. L. Wilson, C. B. Chiu, Phys. Rev. D36 (1987) 1453.

[11] V. M. Braun, prep: hep-ph/9801222 (1998).

[12] V. M. Braun, prep: hep-ph/9911206 (1999).

[13] V. M. Braun, I. E. Filyanov, Z. Phys. C44 (1989) 157.

[14] T. M. Aliev, A. Özpineci, M. Savci, Phys. Rev. D62 (2000) 053012.

[15] V. Chung, H. G. Dosch, M. Kremer, D. Scholl, Nucl. Phys. B197 (1982) 55.

[16] H. G. Dosch, M. Jamin and S. Narison, Phys. Lett. B220 (1989) 251.

[17] I. I. Balitsky, V. M. Braun, Nucl. Phys. B311 (1988) 541.

[18] V. M. Braun, I. E. Filyanov, Z. Phys. C48 (1990) 239.

[19] I. I. Balitsky, V. M. Braun, A. V. Kolesnichenko, Nucl. Phys. B312 (1989) 509.

[20] A. Ali, V. M. Braun, Phys. Lett. B359 (1995) 223.

[21] T. M. Aliev, A. Özpineci, M. Savci, Nucl. Phys. A678 (2000) 443;

T. M. Aliev, A. Özpineci, M. Savci, Phys. Lett. B516 (2001) 299.

[22] V. M. Belyaev, V. M. Braun, A. Khodjamirian and R. Rückl, Phys. Rev. D51 (1995) 6177.

[23] T. M. Aliev, M. Savc1, Phys. Rev. D61 (2000) 0160008. 
[24] I. I. Balitsky, A. V. Kolesnichenko, Yad. Fiz. 41 (1985) 282.

[25] V. M. Belyaev, Ya. I. Kogan, Yad. Fiz. 40 (1984) 1035.

[26] V. M. Belyaev, B. L. Ioffe, JETP 56 (1982) 493.

[27] S. Coleman and S. Glashow, Phys. Rev. Lett. 6 (1961) 423.

[28] B. O. Kerbikov and Yu. A. Simonov, Phys. Rev. D62 (2000) 093016.

[29] S. J. Puglia, M. J. Ramsey-Musolf, Phys. Rev. D62 (2000) 034010.

[30] N. W. Park and H. Weigel, Nucl. Phys. A541 (1992) 453.

[31] Particle Data Group, D. E. Groom at. al, Eur. Phys. J. C15 (2000) 1.

[32] J. Franklin, prep: hep-ph/0103192 (2001).

[33] F. X. Lee and Xinyu Liu, prep: nucl-th/0203051 (2002). 


\section{Figure captions}

Fig. (1) The dependence of the magnetic moment (in units of nuclear magneton $\mu_{N}$ ) of $p$ on the Borel parameter $M^{2}$, at the continuum threshold $s_{0}=2.0 \mathrm{GeV}^{2}$.

Fig. (2) The same as in Fig. (1), but for $n$.

Fig. (3) The same as in Fig. (1), but for $\Lambda$, at the continuum threshold $s_{0}=2.5 \mathrm{GeV}^{2}$ and $m_{s}=0.15 \mathrm{GeV}$.

Fig. (4) The same as in Fig. (3), but for $\Sigma^{0}$, at the continuum threshold $s_{0}=3.0 \mathrm{GeV}^{2}$ and $m_{s}=0.15 \mathrm{GeV}$.

Fig. (5) The same as in Fig. (4), but for $\Sigma^{-}$.

Fig. (6) The same as in Fig. (4), but for $\Sigma^{+}$.

Fig. (7) The same as in Fig. (3), but for $\Xi^{0}$, at the continuum threshold $s_{0}=3.2 \mathrm{GeV}^{2}$ and $m_{s}=0.15 \mathrm{GeV}$.

Fig. (8) The same as in Fig. (7), but for $\Xi^{-}$.

Fig. (9) The dependence of $\lambda_{B}^{2}$ on $t$, for all members of the octet baryons, at the continuum threshold $s_{0}=2.0 \mathrm{GeV}^{2}$ (for $p$ and $n$ ), $s_{0}=2.5 \mathrm{GeV}^{2}$ (for $\Lambda$ ), $s_{0}=3.0 \mathrm{GeV}^{2}$ (for $\Sigma$ ) and $s_{0}=3.2 \mathrm{GeV}^{2}$ (for $\Xi$ ).

Fig. (10) The dependence of the magnetic moments of the octet baryons on $\cos \theta$ at the continuum threshold $s_{0}=2.0 \mathrm{GeV}^{2}$ (for $p$ and $n$ ), $s_{0}=2.5 \mathrm{GeV}^{2}$ (for $\Lambda$ ), $s_{0}=3.0 \mathrm{GeV}^{2}$ (for $\Sigma$ ) and $s_{0}=3.2 \mathrm{GeV}^{2}$ (for $\Xi$ ), and at $M^{2}=1 \mathrm{GeV}^{2}$ (for $p$ and $n$ ), $M^{2}=1.5 \mathrm{GeV}^{2}$ (for $\Sigma$ and $\Lambda$ ) and $M^{2}=1.9 \mathrm{GeV}^{2}$ (for $\Xi$ ). 


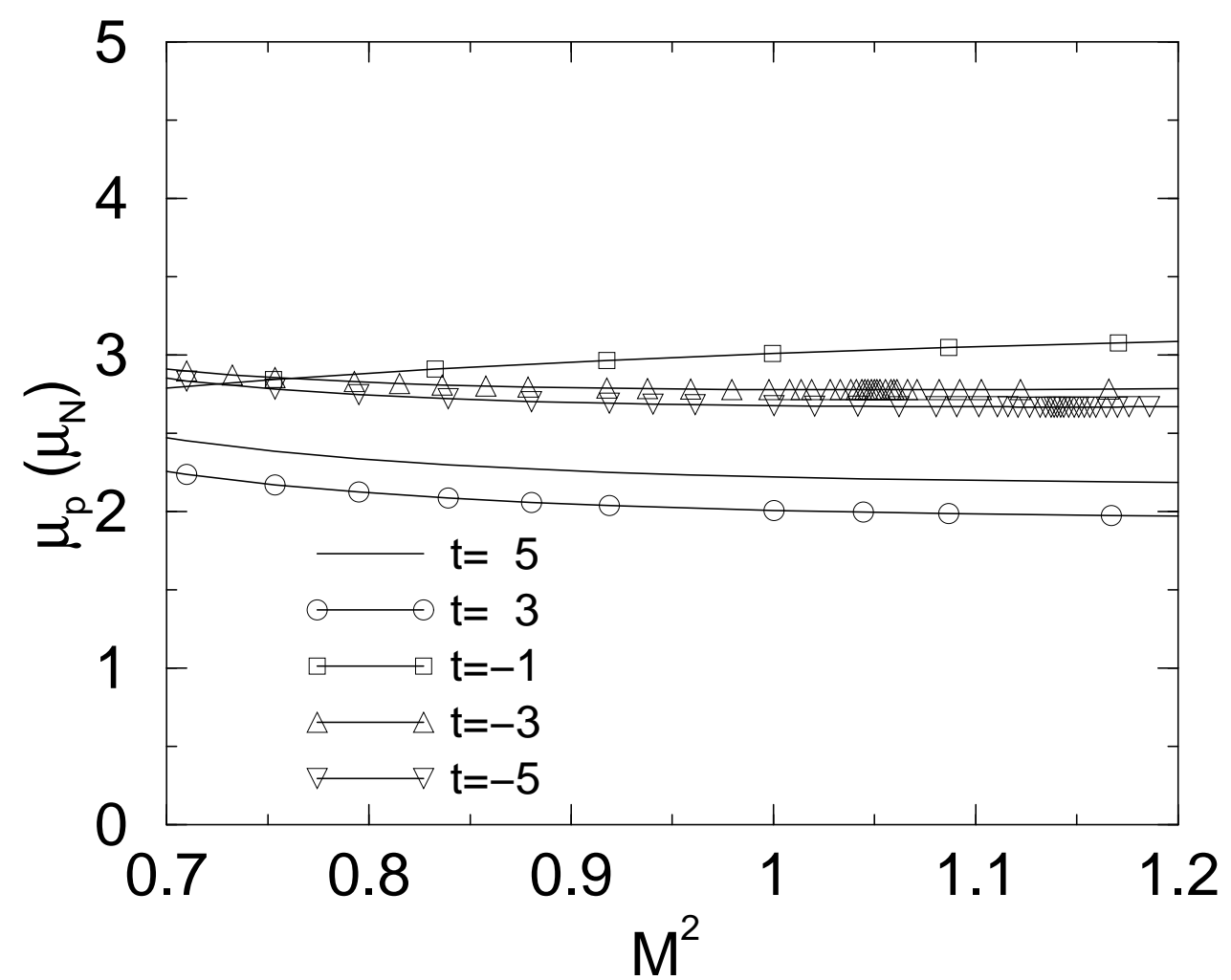

Figure 1:

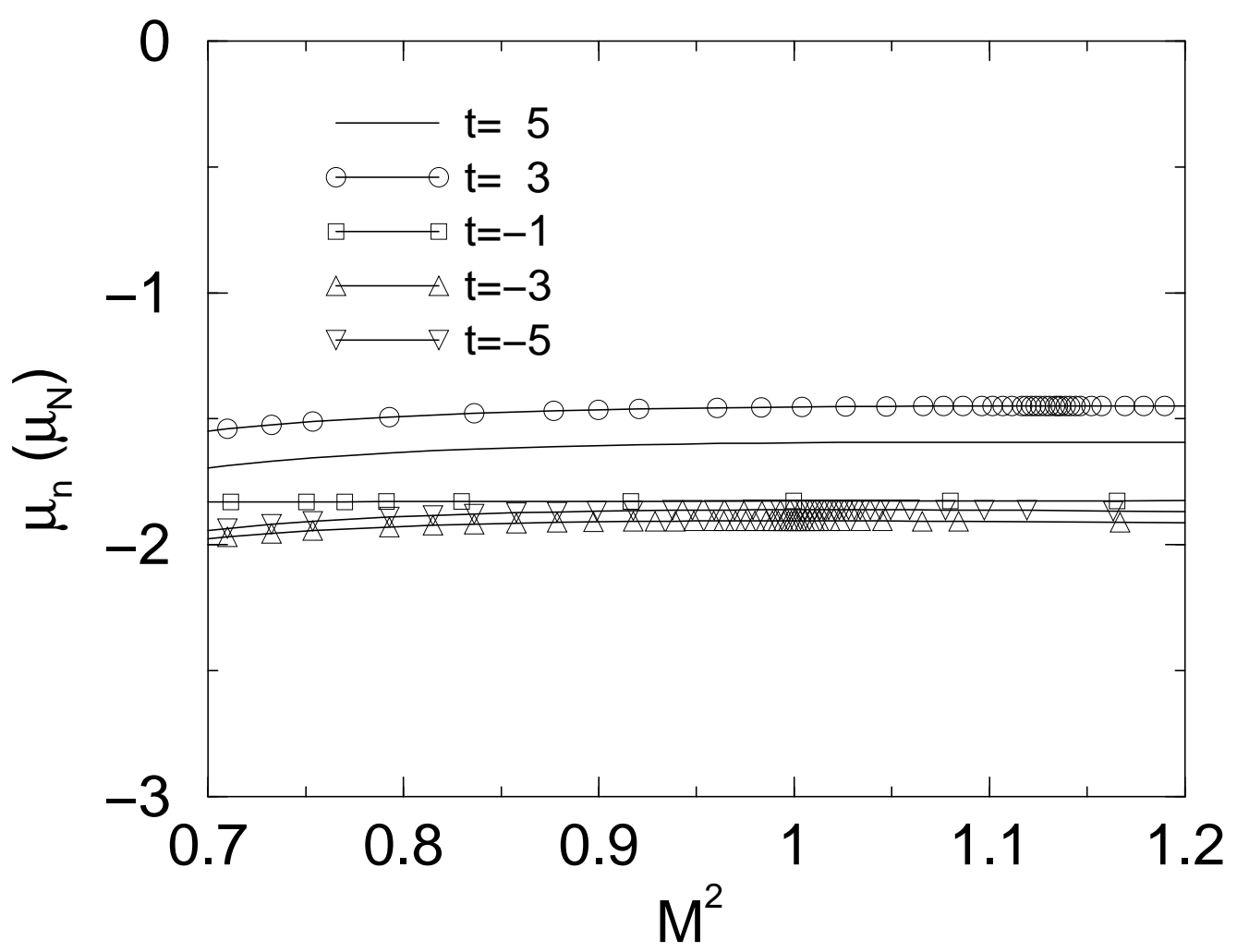

Figure 2: 


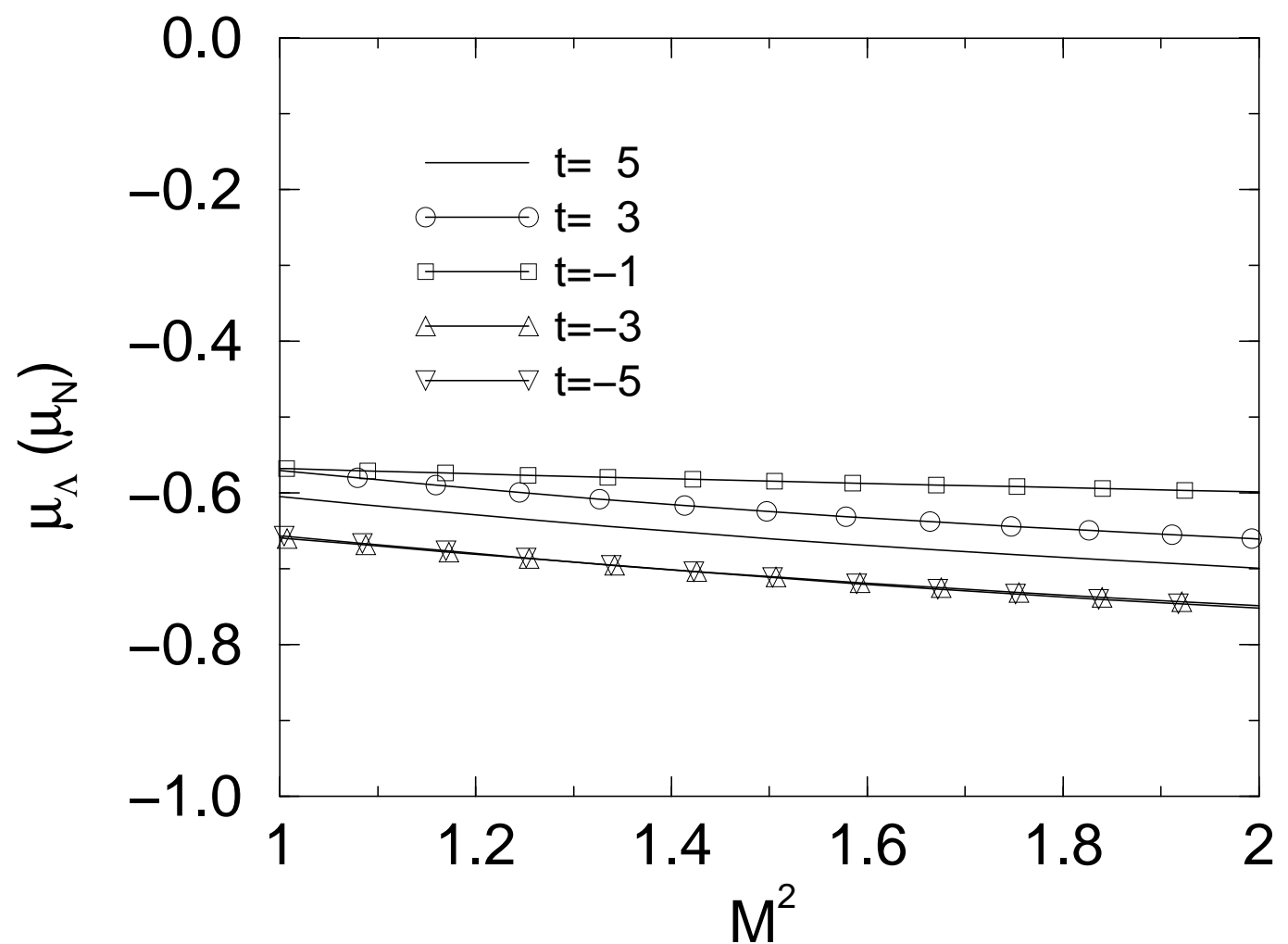

Figure 3:

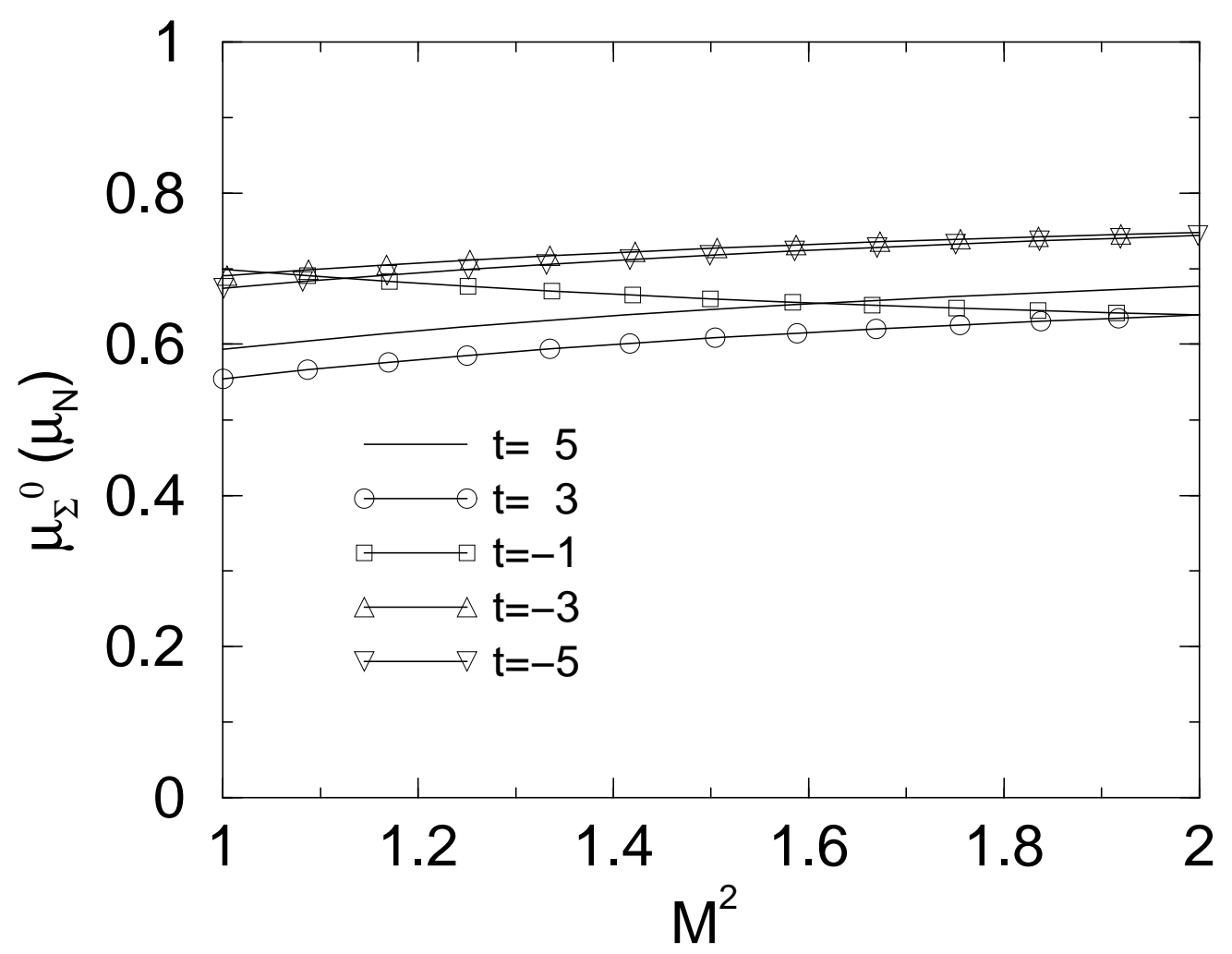

Figure 4: 


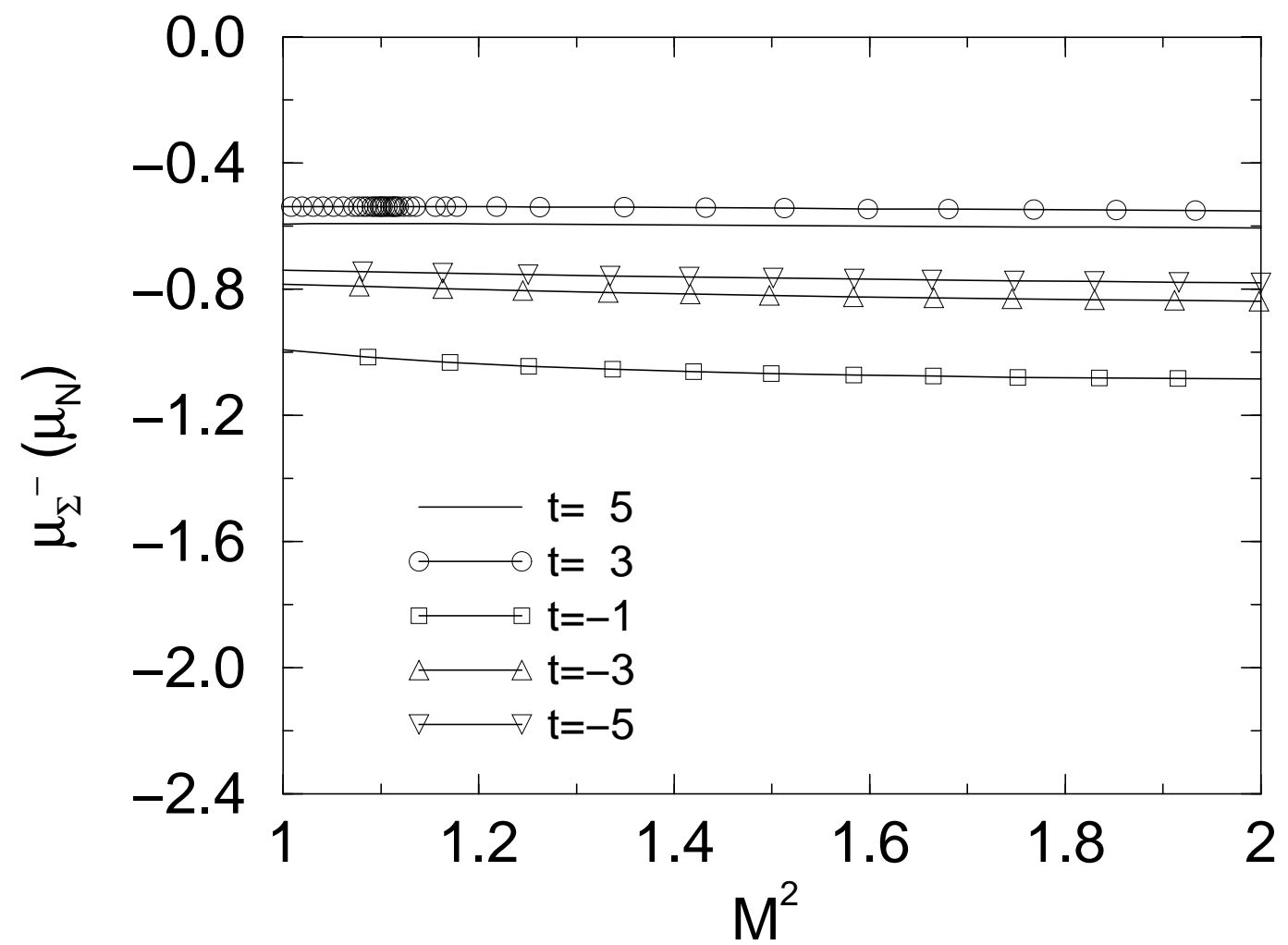

Figure 5:

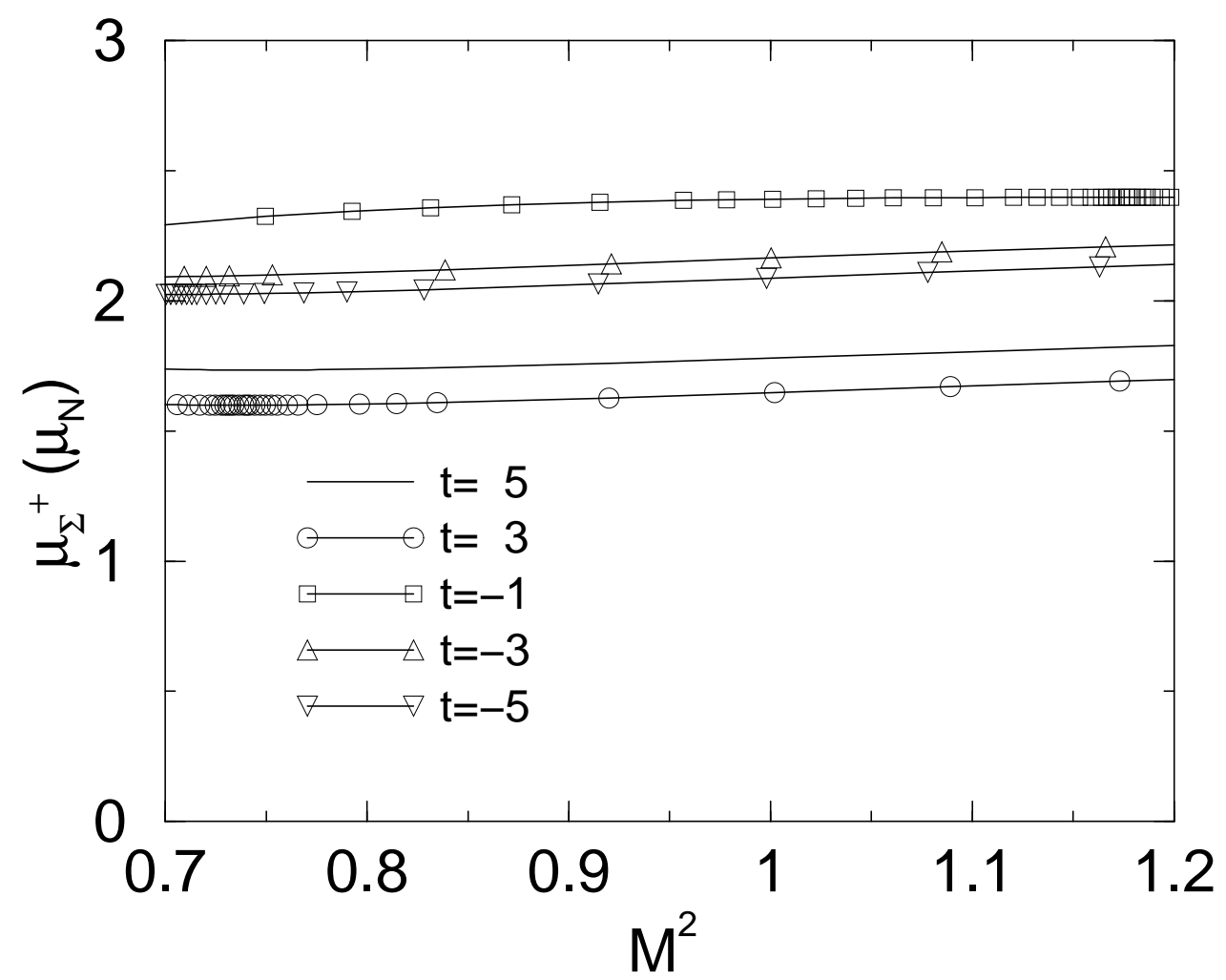

Figure 6: 


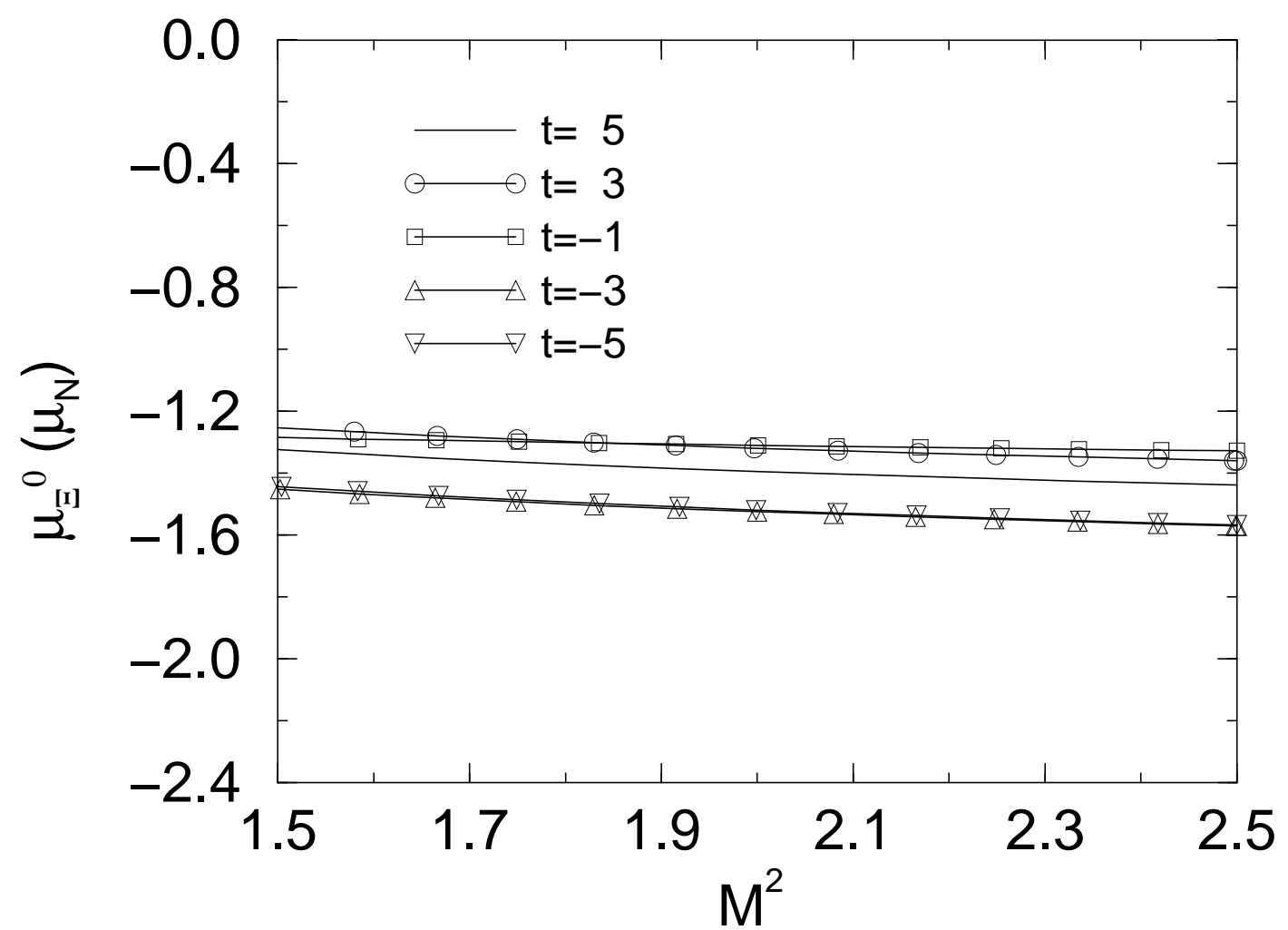

Figure 7:

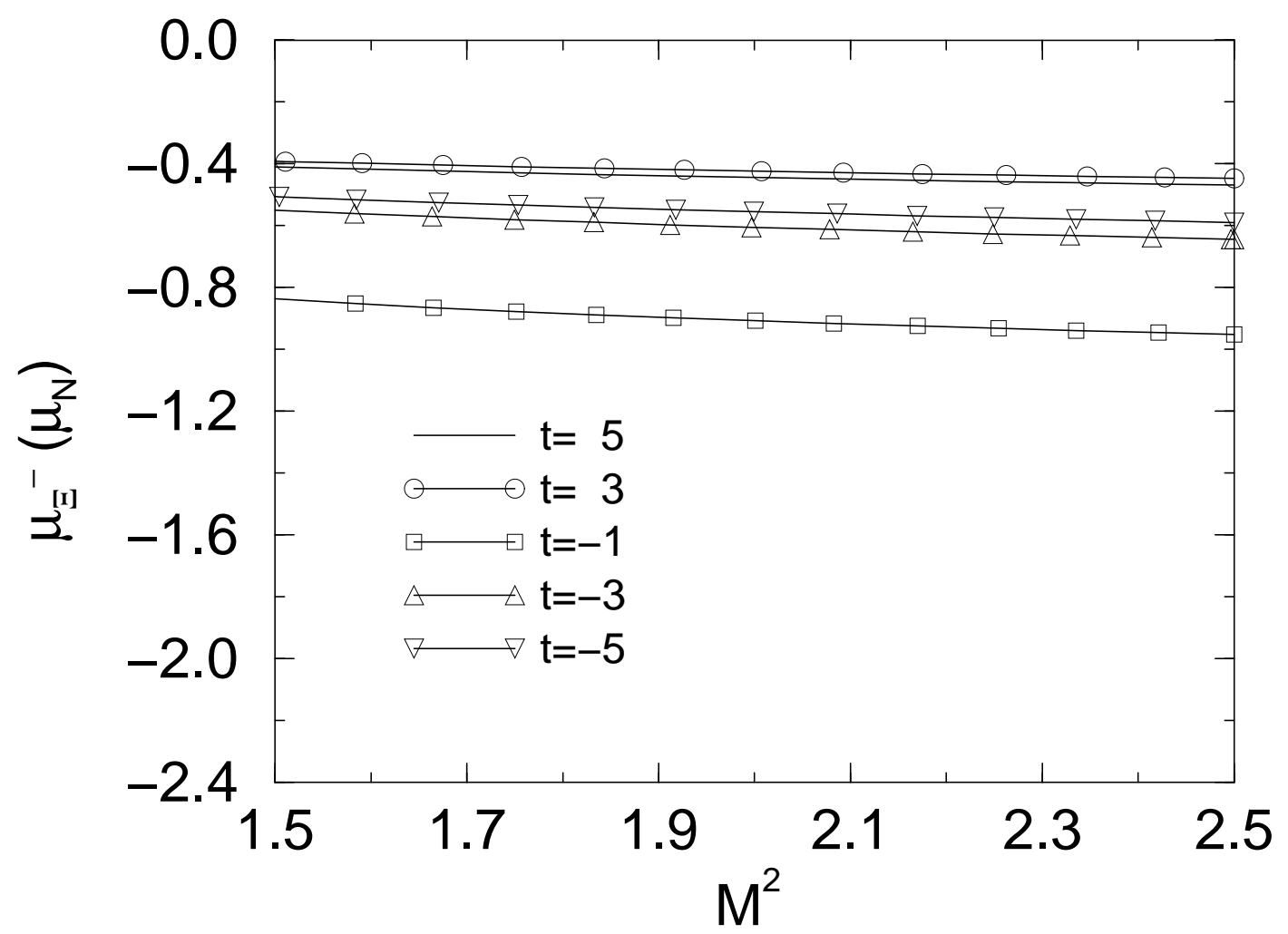

Figure 8: 


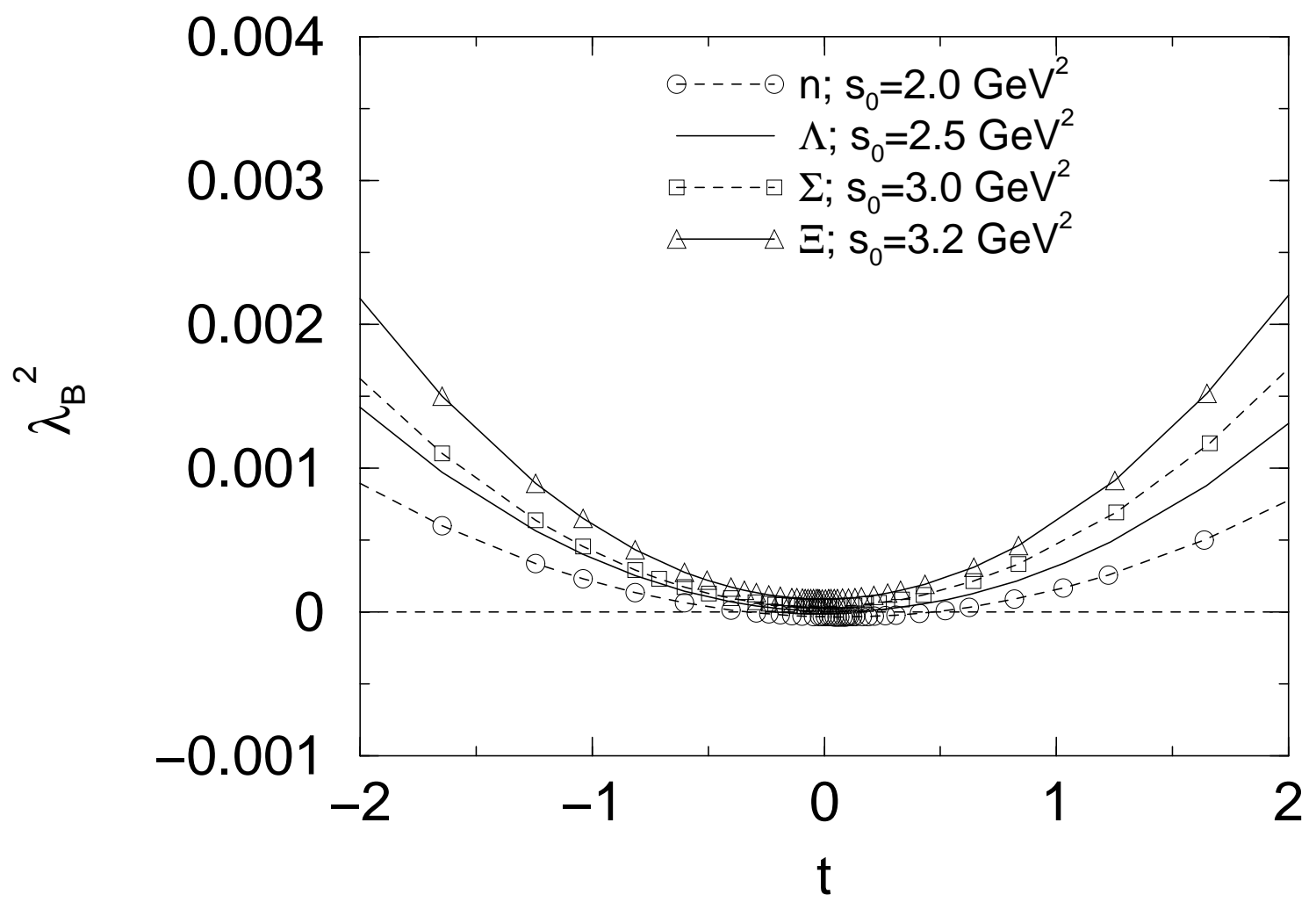

Figure 9:

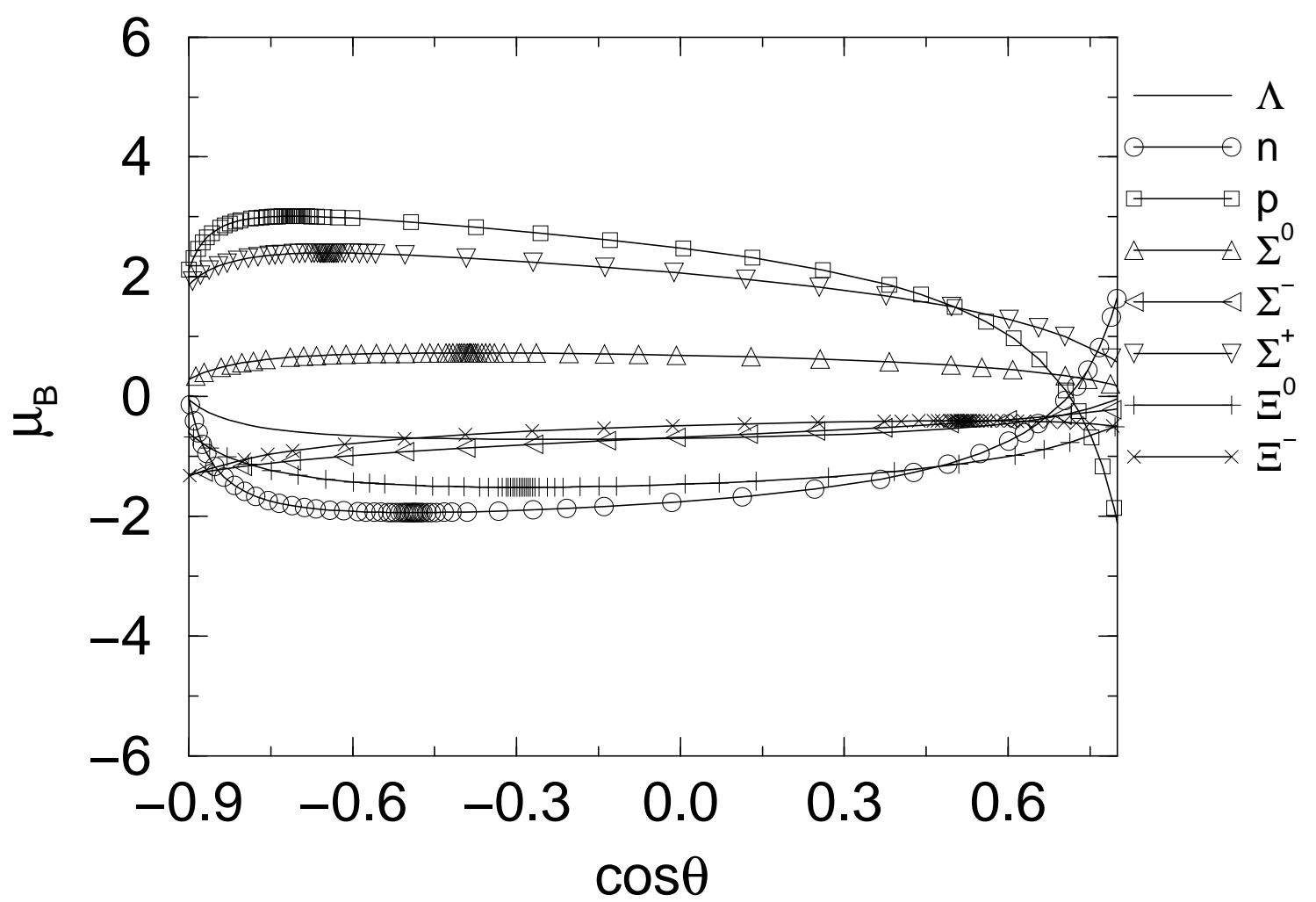

Figure 10: 